\title{
BMJ Open Protocol for the China PEACE (Patient- centered Evaluative Assessment of Cardiac Events) Million Persons Project pilot
}

\author{
Jiapeng Lu, ${ }^{1}$ Si Xuan, ${ }^{2,3}$ Nicholas S Downing, ${ }^{2}$ Chaoqun Wu, ${ }^{1} \mathrm{Li} \mathrm{Li},{ }^{1}$ \\ Harlan M Krumholz, ${ }^{2,3,4}$ Lixin Jiang $^{1}$
}

To cite: Lu J, Xuan S, Downing NS, et al. Protocol for the China PEACE (Patientcentered Evaluative Assessment of Cardiac Events) Million Persons Project pilot. BMJ Open 2016;6:e010200. doi:10.1136/bmjopen-2015010200

- Prepublication history and additional material is available. To view please visit the journal (http://dx.doi.org/ 10.1136/bmjopen-2015010200).

$\mathrm{JL}$ and $\mathrm{SX}$ are the co-first authors. HMK and LJ are joint senior authors.

Received 6 October 2015 Revised 10 November 2015 Accepted 26 November 2015

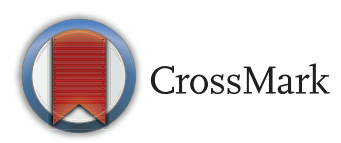

For numbered affiliations see end of article.

Correspondence to Professor Lixin Jiang; jiangl@fwoxford.org

\section{ABSTRACT}

Introduction: Collection of high-quality data from large populations is considered essential to generate knowledge that is critical to an era of precision medicine. Cardiovascular disease (CVD) is a leading cause of mortality in China and is a suitable focus of an initiative to discover factors that would improve our ability to assess and modify individual risk.

Methods and analysis: The pilot phase of China PEACE (Patient-centered Evaluative Assessment of Cardiac Events) Million Persons Project is being conducted during 2014-2015 in four provinces across China to demonstrate the feasibility of a populationbased assessment. It is designed to screen 0.4 million community-dwelling residents aged $40-75$ years with measurements of blood pressure, height and weight, a lipid blood test, and a questionnaire on cardiovascularrelated health status. Participants identified at high risk of CVD receive further health assessments, including ECG, ultrasound scan, blood and urine analysis, and a questionnaire on lifestyle and medical history. Collection of blood and urine samples is used to establish a biobank. High-risk subjects are also counselled with suggestions regarding potential lifestyle changes. In addition, high-risk subjects are followed-up either in a return clinic visit or by telephone interview, with measurement of blood pressure, weight, ECG, and a questionnaire on survival status, hospitalisations and lifestyle. The first 0.1 million participants screened were used to conduct a preliminary analysis, with information on baseline characteristics, health-related behaviours, anthropometric variables, medical history, and prevalence of high-risk subjects.

Ethics and dissemination: The central ethics committee at the China National Center for Cardiovascular Disease (NCCD) approved the pilot. Written informed consent is obtained from all participants on entry into the project. Findings will be disseminated in future peer-reviewed papers and will inform strategies aimed at developing precise methods of assessing and modifying risk.

Trial registration number: NCT02536456.

\section{Strengths and limitations of this study}

- The pilot is the first large-scale population-based screening project in China aimed at identifying subjects at high risk of cardiovascular disease (CVD) and collecting detailed information and biospecimens as part of a precision medicine project.

- With rigorous methodological design and data collection, this public health effort can serve as a powerful research-grade database for future precision medicine investigations into the biological, environmental, behavioural and other contextual factors associated with CVD in the Chinese population.

- The pilot project was conducted primarily to test the feasibility of a large-scale screening project, and the integrated quality assurance procedures ensure its ability to act as a reliable resource for future research.

- Insights garnered from this project will inform approaches for future efforts in developing individualised approaches to primary and secondary CVD prevention in China.

\section{INTRODUCTION}

A central challenge in medicine is to individualise approaches to patient treatment. However, much of medicine is based on study results of averages for populations, and there is a general lack of knowledge about how best to individualise strategies. Precision medicine is a term that refers to efforts to better understand individual differences and to tailor clinical care for each person in a more customised way. ${ }^{1}$ To generate knowledge about individuals requires studies of massive numbers of people, so that those with similar characteristics can be studied and their risks understood.

China is an ideal country to undertake such studies because of its large population, and cardiovascular disease (CVD), a major 
public health challenge in China, is a suitable condition for the focus of such an initiative. Few Chinese adults have ideal cardiovascular health, ${ }^{2}$ and the increasing prevalence of hypertension, ${ }^{3}$ diabetes, ${ }^{4}$ smoking ${ }^{5}$ and obesity, ${ }^{6}$ combined with an aging population, ${ }^{7}$ is likely to result in increasing numbers of CVD events in the years to come. For example, it is predicted that CVD events in China will increase by more than one half of their current value over the next two decades, ${ }^{8}$ and that there could be as many as 20 million myocardial infarctions (MIs) and 30 million strokes per year by $2030 .^{9}$

Risk factor modification has the potential to prevent CVD and is broadly recommended by international guidelines for the prevention of CVD. ${ }^{10}{ }^{11}$ Evidence from randomised controlled trials has shown that lipid-lowering treatments using statin ${ }^{12-15}$ and antihypertensive drugs ${ }^{16}{ }^{17}$ may reduce the incidence and mortality rates of CVD. In addition to changes in medication, lifestyle interventions such as smoking cessation, ${ }^{18}$ increased physical activity ${ }^{19} 20$ and dietary improvements $^{21-23}$ are also associated with lower rates of incidence of CVD. However, the value of preventive interventions depends on an individual's cardiovascular risk. ${ }^{24-26}$ Therefore, efficient identification of those at high CVD risk is necessary for the proper implementation of disease-prevention strategies.

Unfortunately, many patients in China have CVD risk factors that remain undiagnosed and uncontrolled. For example, a cross-sectional national survey of Chinese adults estimated that $70 \%$ of diabetes cases were undiagnosed, and even those that were diagnosed were commonly not well controlled, especially in underdeveloped regions. ${ }^{27}$ This pattern was also common in cases of dyslipidaemia and hypertension. ${ }^{28}{ }^{29}$ To date, there has been no large-scale, population-based screening study implemented in China to identify and counsel subjects with high CVD risk. Knowledge regarding Chinese adults with high CVD risk is also limited; very few studies have recognised high-risk rural patients. ${ }^{30}$

Consequently, the Chinese government has committed to the China PEACE (Patient-centered Evaluative Assessment of Cardiac Events) Million Persons Project (MPP), the pilot protocol of which we report here. China PEACE is an administrative structure to support the generation and implementation of studies to improve the care and outcomes of patients in Chinaand produce knowledge that will help people around the world. China PEACE MPP is a population-centred national screening initiative to detect populations at high risk of GVD. It will collect biospecimens and detailed information on sociodemographics, disease histories, extreme phenotypes, lifestyles, and behaviours for millions of people. Previously published China PEACE studies have focused primarily on care and outcomes for patients who have had acute MI and/or percutaneous coronary intervention (PCI). ${ }^{31}{ }^{32}$ Moving beyond such hospital-based studies, China PEACE MPP will add knowledge about broader population-based cardiovascular health and generate important insights into primary and secondary prevention of CVD in China. Our work is similar in nature to the Precision Medicine Initiative (PMI) launched in the USA in January 2015, which aims to recruit 1 million Americans and "will encourage and support the next generation of scientists to develop creative new approaches for detecting, measuring, and analysing a wide range of biomedical information-including molecular, genomic, cellular, clinical, behavioural, physiological, and environmental parameters. ${ }^{, 33}$ Like the PMI, our national screening initiative will collect biological samples and lifestyle information from a voluntary national research cohort to create a platform for precision medicine studies. Using collected information, researchers will be able to investigate individual differences in genes, behaviours and lifestyles to advance the emerging field of precision medicine. The US PMI will put a near-term focus on supporting more and better cancer treatments, whereas our study will focus on cardiovascular health. Further investigation of cardiovascular health is especially important in a country such as China, which has an aging population and is increasingly urbanised. Our initiative should, in the long run, provide important insights in informing future efforts to develop more individualised approaches to CVD prevention and intervention. In the near-term, the public health component of this initiative seeks to address the nation's pressing needs to identify high-risk CVD subjects, characterise population risk factors, and define the relationship between risk factors and CVD outcomes in high-risk populations.

This effort, funded by the Ministry of Finance (MOF) of China and the National Health and Family Planning Commission (NHFPC) of China, was initially planned for 0.4 million people across four provinces, with the potential for expansion to 4 million across the country. Thus, the China PEACE MPP Pilot is designed to determine if a much-needed and large-scale public health effort could be feasibly paired with a high-quality research programme to amass a database capable of supporting and advancing precision medicine research in China. The pilot launched in 2014 is using methodologies that include a rigorous protocol design, standardised operational features, data collection methods capable of promoting future investigation, and the establishment of a biobank. The goal of the pilot is to determine the feasibility of a subsequent future nationwide screening project and will also generate data for future research on the understanding and prevention of CVD.

\section{METHODS}

\section{Design overview}

The pilot of the China PEACE-MPP began in July 2014 and has continued into 2015. The pilot project is taking place in four provinces, which were chosen according to their local available human resources and capacity to perform a large-scale screen. About 0.4 million residents 
are being screened using measurements of blood pressure, height and weight, a lipid blood test, and a questionnaire assessing cardiovascular-related health status. This process aims to identify subjects with high CVD risk, where 'high CVD risk' is defined as meeting one of the following criteria: (1) history of established CVD; (2) high blood pressure; (3) dyslipidaemia; (4) a 10-year risk of CVD $\geq 20 \%$. ${ }^{34}$ These high-risk subjects then receive a detailed assessment of their cardiovascular health based on information collected from an ECG, ultrasound scans, biosampling, laboratory tests, and an extended questionnaire assessing lifestyle (eg, smoking, physical activity, diet) and medical history. In addition, the high-risk subjects are counselled with suggestions regarding potential lifestyle changes (eg, smoking cessation, increasing physical activity, weight loss). High-risk subjects are followed-up either in a return clinic visit or through a telephone interview. In the pilot, we conducted a 1-month follow-up to test if a high rate for such a large-scale follow-up could be achieved. The follow-up assessment consists of blood pressure and weight measurements, an ECG, and a questionnaire assessing cardiovascular health status.

The central ethics committee at the China National Center for Cardiovascular Disease (NCCD) approved the pilot. The Chinese government, which provides financial support, has had no role in the design or administration of the study, the collection, management, analysis or interpretation of the data, or the preparation or approval of this paper. Written informed consent is obtained from all participants on entry into the project. The informed consent form states that all personal information and any results from the physical measurements, laboratory tests and other tests are confidential and stored in an encrypted database. Knowledge generated from this project will be disseminated in future peerreviewed papers and will inform strategies aimed at developing precise prevention and intervention methods for CVD.

\section{Pilot sites and recruitment}

The pilot is conducted in 20 geographically defined regions (11 urban districts and 9 rural counties) of four provinces (Jilin, Liaoning, Zhejiang and Guangxi) in China (figure 1). The regions were selected on the basis of their geographic location, quality of disease and death registries, and local capacity to support the pilot. In each region, 3-5 urban residential communities or rural villages were chosen according to community or village size, population stability (eg, no sudden significant change in the number of residents), and commitment and ability of local workers to perform the screening. Initial screening stations were set up in each community or village health centre. Subjects identified as being at high risk of CVD at a screening station are then moved to a designated hospital within each region to receive further assessment, counselling and follow-up care. Each hospital is selected on the basis of its distance from local residents and its ability to perform laboratory tests, ECGs, ultrasound scans and health counselling.

Potentially eligible participants are identified in each community or village through official residential records, and then invited by local community workers via extensive publicity campaigns on the television and in the newspapers. The participant response rate for each community or village will be related to its known populations. All participants are required to bring their identity cards to the screening centre to verify that they meet both of the inclusion criteria: (1) aged 40-75; (2) registered in the Hukou (a record officially identifying a person as a resident of an area) of the selected region. The qualifying age range of 40-75 years was chosen according to the WHO/International Society of Hypertension (ISH) cardiovascular risk prediction charts. ${ }^{34}$ After verification of residency, participants who have signed the informed consent agreement (see online supplementary appendix 1) are then enrolled for initial screening.

\section{Initial screening protocol}

Participants are first asked for sociodemographic information (eg, education, income, health insurance; see online supplementary appendix 2 ). They then receive a physical examination, a lipid blood test and an in-person interview performed by trained medical staff. The whole screening process takes about 30 min to complete.

\section{Physical measurements}

For each participant, blood pressure is measured on the right upper arm after 5 min of rest in a seated position using an electronic blood pressure monitor (Omron HEM-7430; Omron Corporation, Kyoto, Japan). Blood pressure is measured twice, with $1 \mathrm{~min}$ between the measurements. The two readings are then recorded and their mean value is calculated in order to identify people at high CVD risk. If the difference between the two systolic blood pressure (SBP) readings is larger than $10 \mathrm{mmHg}$, a third blood pressure measurement is taken, and the mean value of the last two readings is calculated. Heart rate readings are also collected using the electronic blood pressure monitor. Participants are required to wear light clothes, no shoes and no cap when trained technicians measure their height and weight. Weight is measured to the nearest $0.1 \mathrm{~kg}$, and height is measured to the nearest $0.1 \mathrm{~cm}$. Body mass index (BMI) is calculated by dividing weight in kilograms by height in metres squared.

\section{Lipid blood test}

A non-fasting lipid blood test that measures total cholesterol (TC), triglyceride (TG), high-density lipoprotein cholesterol (HDL-C) and low-density lipoprotein cholesterol (LDL-C) is performed by a rapid lipid analyser using fingertip blood samples (CardioChek PA Analyzer; Polymer Technology Systems, Indianapolis, Indiana, USA). This device uses reflected light to measure an 


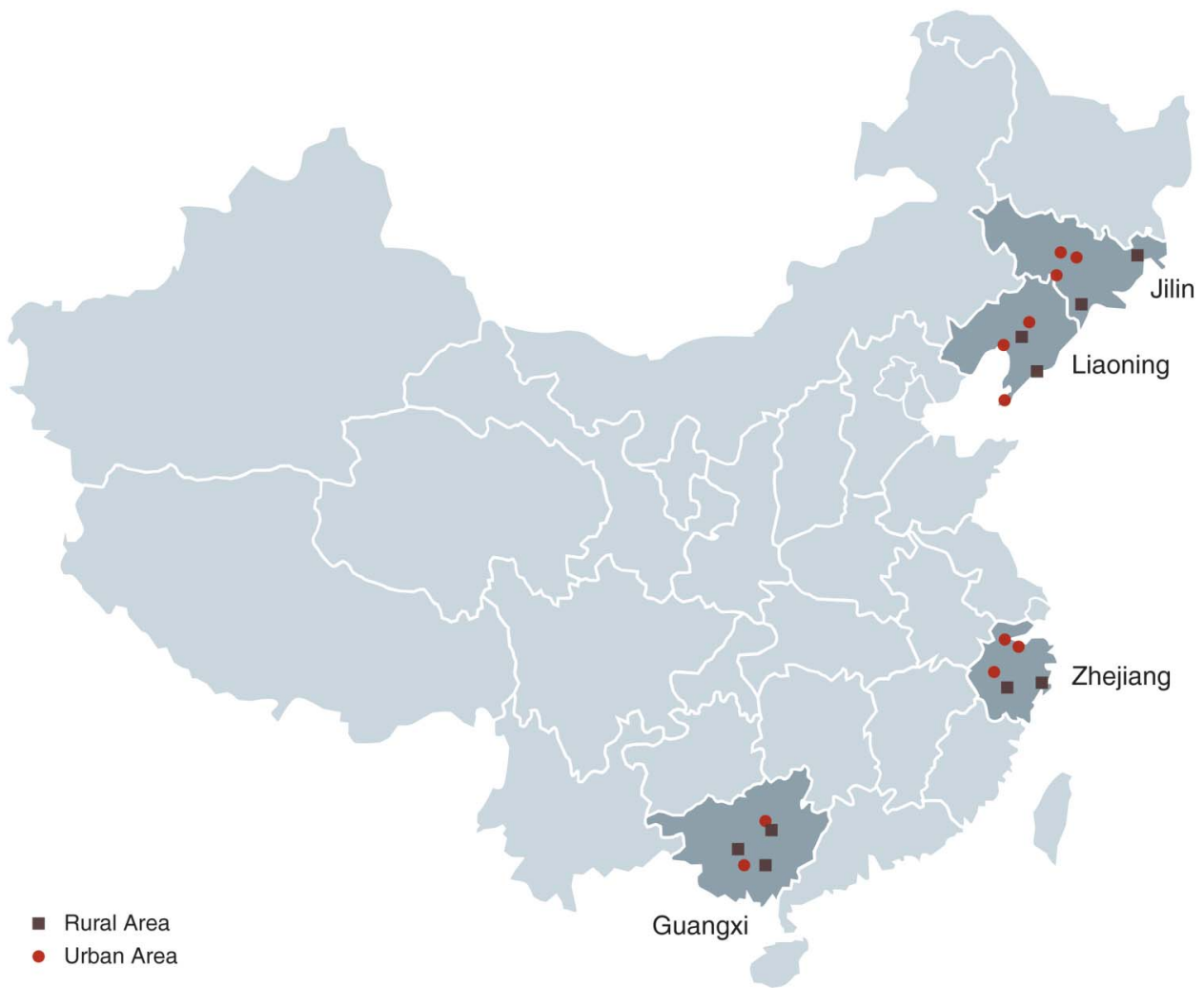

Figure 1 Geographic distribution of pilot sites. The pilot sites are located in 20 geographically defined regions from four provinces (Jilin, Liaoning, Zhejiang, Guangxi) in China. The 20 local regions consist of 11 urban districts and 9 rural counties.

end point enzymatic chemical reaction. The lipid panel test strips are designed for testing of $\mathrm{TC}, \mathrm{TG}$ and HDL-C. LDL-C is calculated using the values of TC, HDL-G and TG levels in the Friedewald equation. A daily quality control check of the device is performed using the grey check strip test, which verifies the accuracy of the device's electronic and optical systems.

\section{Initial questionnaire on cardiovascular health status}

After the physical measurements and lipid blood test are completed, participants have a 5-10 min in-person interview with a trained staff member using a computerdelivered questionnaire (see online supplementary appendix 3). In order to identify high-risk subjects, the questionnaire assesses: cigarette smoking status; alcohol consumption; self-reported history of hypertension, diabetes, MI, PCI, coronary artery bypass grafting (CABG), and stroke; self-reported history of medication; and extreme phenotypes (as indicated by family history of longevity, premature death and prevalent chronic diseases). Participants are classified as current smokers if they answer, 'Yes' to the question, 'Do you currently smoke cigarettes?' A full list of variables is given in table 1 .

A written health report including the results of the physical measurements and the lipid blood test is given to each initial screening participant (see online supplementary appendix 4).

\section{Identification of high-risk subjects}

Participants are considered at high risk of CVD if they meet at least one of four criteria (figure 2). The criteria are adapted from WHO guidelines for the assessment and management of cardiovascular risk. ${ }^{34}$ In recognition of the lower lipid levels found in the Chinese population, the WHO lipid criteria were adjusted so that more individuals could be classified as high-risk subjects and the relationship between more modestly elevated lipid levels and CVD risk could be determined. The four criteria for eligibility for the pilot are:

1. History of at least one of the following cardiovascular events

MI, PCI, CABG treatment, or stroke (either ischaemic or haemorrhage)

2. High blood pressure

Defined as $\mathrm{SBP} \geq 160 \mathrm{mmHg}$ or diastolic blood pressure $(\mathrm{DBP}) \geq 100 \mathrm{mmHg}$

3. Dyslipidaemia

Defined as LDL-C $\geq 160 \mathrm{mg} / \mathrm{dL}(4.14 \mathrm{mmol} / \mathrm{L})$ or $\mathrm{HDL}-\mathrm{C}<30 \mathrm{mg} / \mathrm{dL}(0.78 \mathrm{mmol} / \mathrm{L})$

4. Risk of CVD in 10 years $\geq 20 \%$ based on WHO/ISH Cardiovascular Risk Prediction Charts for the Western Pacific Region $\mathrm{B}^{35}$

Risk is determined using the following information: age, gender, smoking status, presence or absence of diabetes, SBP and TC. The risk of CVD is calculated by a predetermined algorithm derived 
Table 1 Information collected in the pilot project

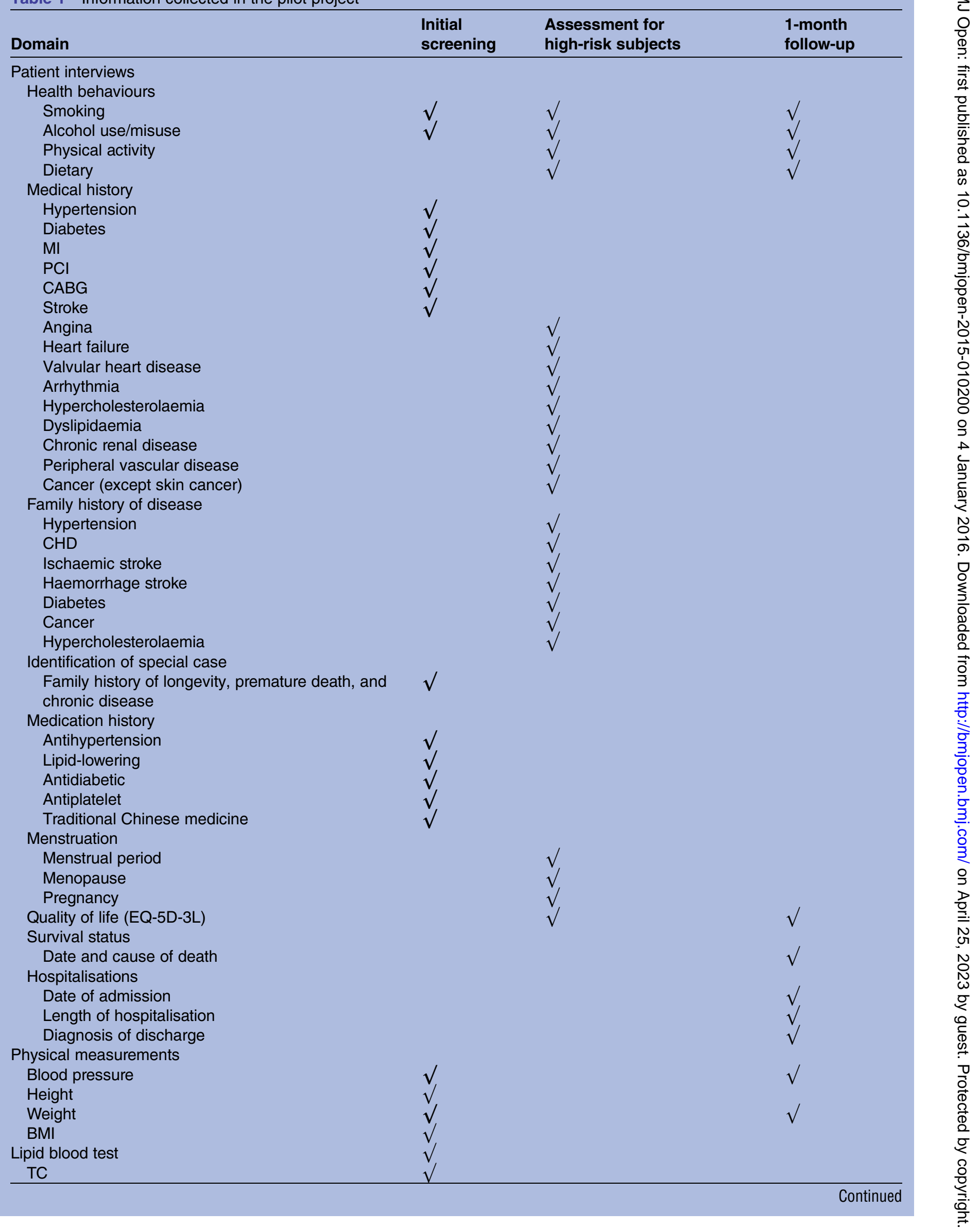


Table 1 Continued

\begin{tabular}{|c|c|c|c|}
\hline Domain & $\begin{array}{l}\text { Initial } \\
\text { screening }\end{array}$ & $\begin{array}{l}\text { Assessment for } \\
\text { high-risk subjects }\end{array}$ & $\begin{array}{l}\text { 1-month } \\
\text { follow-up }\end{array}$ \\
\hline TG & $\sqrt{ }$ & & \\
\hline LDL-C & $\sqrt{ }$ & & \\
\hline HDL-C & $\sqrt{ }$ & & \\
\hline \multicolumn{4}{|l|}{ Imaging examinations } \\
\hline$E C G$ & & $\sqrt{ }$ & $\sqrt{ }$ \\
\hline Echocardiogram & & $\sqrt{ }$ & \\
\hline Carotid artery ultrasound & & $\sqrt{ }$ & \\
\hline \multicolumn{4}{|l|}{ Biosamples } \\
\hline Blood & & $\sqrt{ }$ & \\
\hline Urine & & $\sqrt{ }$ & \\
\hline \multicolumn{4}{|l|}{ Laboratory analysis } \\
\hline \multicolumn{4}{|l|}{ Biochemistry test } \\
\hline Blood lipid & & $\sqrt{ }$ & \\
\hline Glucose & & $\sqrt{ }$ & \\
\hline ALT & & $\sqrt{ }$ & \\
\hline AST & & $\sqrt{ }$ & \\
\hline Creatinine & & $\sqrt{ }$ & \\
\hline Uric acid & & $\sqrt{ }$ & \\
\hline $\mathrm{HbA1c}$ & & $\sqrt{ }$ & \\
\hline \multicolumn{4}{|l|}{ Urine routine test } \\
\hline Glucose & & $\sqrt{ }$ & \\
\hline Ketone & & $\sqrt{ }$ & \\
\hline Occult blood & & $\sqrt{ }$ & \\
\hline Protein & & $\sqrt{ }$ & \\
\hline Nitrite & & $\sqrt{ }$ & \\
\hline Bilirubin & & $\sqrt{ }$ & \\
\hline Gravity & & $\sqrt{ }$ & \\
\hline $\mathrm{pH}$ & & $\sqrt{ }$ & \\
\hline Urobilinogen & & $\sqrt{ }$ & \\
\hline Erythrocyte & & $\sqrt{ }$ & \\
\hline Leucocyte & & $\sqrt{ }$ & \\
\hline
\end{tabular}

ALT, alanine aminotransferase; AST, aspartate aminotransferase; BMI, body mass index; CABG, coronary artery bypass grafting; CHD, coronary heart disease; ECG, 12-lead ECG; HbA1c, glycated haemoglobin; HDL-C, high-density lipoprotein cholesterol; LDL-C, low-density lipoprotein cholesterol; MI, myocardial infarction; PCI, percutaneous coronary intervention; TC, total cholesterol; TG, triglyceride.

from the $\mathrm{WHO} / \mathrm{ISH}$ cardiovascular risk prediction charts for the Western Pacific Region B. $^{35}$ Individuals with $\geq 20 \%$ risk of CVD are considered to be high-risk subjects. ${ }^{34}$

\section{Health assessment of high-risk subjects}

Following their initial screening, all high-risk subjects receive a detailed assessment of their cardiovascular health based on physical measurements, biosamples, laboratory tests and an extended questionnaire.

\section{Physical measurements}

Trained medical staff members from local hospitals perform a 12-lead ECG using a multichannel ECG machine linked to an ECG interpretation and transfer computer system (HW-E100; Hanwei Medical Group, Hebei, China). Local ultrasound physicians then conduct echocardiography and a carotid artery ultrasound scan in accordance with standards set down by the NHFPC, China. Trained ultrasound physicians interpret and provide the written results of the echocardiogram and the carotid artery ultrasound scan to high-risk subjects (see online supplementary appendices 5 and $6)$. The quality control team, which consists of the senior ultrasound physicians from that local site, monitors medical staff compliance with ultrasound protocols on a daily basis. Every day, they randomly select $5 \%$ of all ultrasound images to determine whether any data entry errors have occurred. Echocardiography and carotid artery ultrasound images are stored at local sites and transmitted on a monthly basis to the NCCD using an encrypted hard disk in Digital Imaging and Communications in Medicine (DICOM) format. ${ }^{36}$ For some rural hospitals that cannot provide DICOM files, static images are stored in JPG format, and dynamic images are stored in a video format such as AVI. Trained ultrasound physicians from the NCCD verify the results of the ECGs and ultrasound scans once those images have been transmitted to the NCCD. 


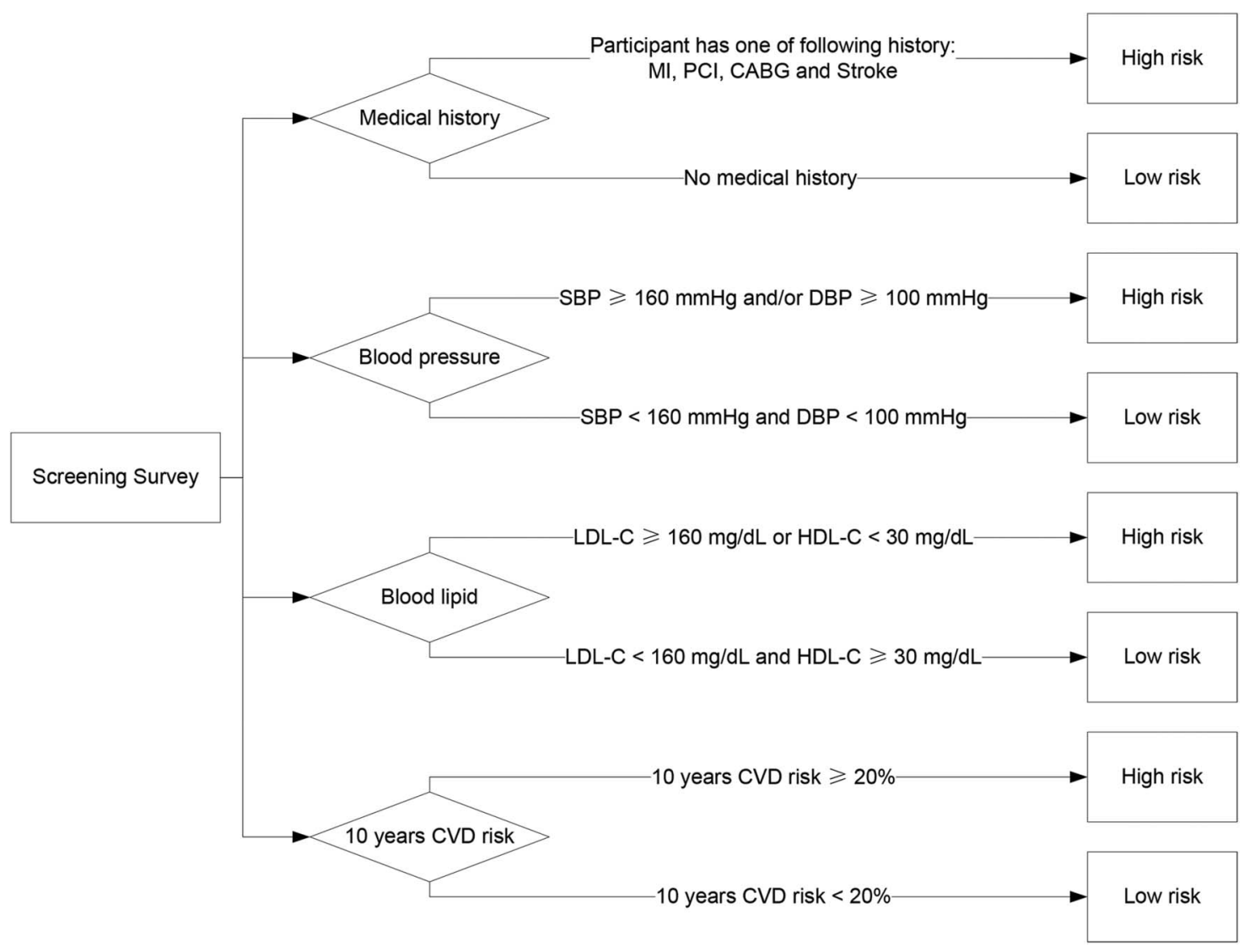

Figure 2 Criteria for identification of high-risk subjects.

\section{Biosamples}

For each high-risk patient, a 5 or $6 \mathrm{~mL}$ whole blood sample is collected in an EDTA vacuum tube in order to test for haemoglobin A1c (HbA1C). A second 5 or $6 \mathrm{~mL}$ whole blood sample is drawn into a serum gel tube for analysis of the biochemical values of the serum. A $10 \mathrm{~mL}$ urine sample is also collected. Within $24 \mathrm{~h}$ of collection, the blood samples are divided into aliquots and centrifuged at $2100 \mathrm{~g}$ for $10 \mathrm{~min}$. The plasma, serum and urine samples are then pipetted into $2 \mathrm{~mL}$ cryovials. All filled cryovials and EDTA vacuum tubes are immediately stored at $-40^{\circ} \mathrm{C}$ or $-80^{\circ} \mathrm{C}$, then transported to the NCCD within 1 month and stored at $-80^{\circ} \mathrm{C}$ or $-180^{\circ} \mathrm{C}$ for central calibration analysis and long-term storage.

\section{Laboratory tests}

A $1 \mathrm{~mL}$ sample of serum is used to perform a biochemistry test measuring blood lipid, glucose, alanine aminotransferase (ALT), aspartate aminotransferase (AST), creatinine and uric acid levels. The HbAlc value is determined via the ionic-exchange high-performance liquid chromatography method (VARIANT II Haemoglobin Testing System; Bio-Rad Laboratories, Hercules, California, USA). In addition, the urine sample is used to conduct a urine routine test measuring glucose, ketone, occult blood, protein, bilirubin and leucocyte levels.
Extended questionnaire on cardiovascular health status

After the physical measurements and laboratory tests, high-risk subjects take part in an extended in-person interview. The interviewer-administered questionnaire includes the following eight topics (see online supplementary appendix 7): smoking (eg, frequency, tobacco type) $;{ }^{37}$ alcohol use/misuse (eg, frequency, dependence symptoms; assessed using the Alcohol Use Disorders Identification Tool (AUDIT)) $;^{38}$ physical activity (eg, activities available in urban or rural locations, exercise level in leisure time) $;{ }^{37}$ diet (eg, frequency of rice, meat, or vegetable consumption) $;{ }^{37}$ personal medical history; family medical history; menstruation and pregnancy history ${ }^{39}$ and quality of life (assessed using the EQ-5D-3L $\left.{ }^{40}\right)$. Questions were adapted from prior population-based epidemiological studies in China. ${ }^{3741}$ The validity and reliability of AUDIT and EQ-5D-3L applied to the Chinese population have been previously evaluated. ${ }^{38} 40$ A full list of variables is shown in table 1.

A written report on the results of the further assessment of high-risk subjects is given to each participant (see online supplementary appendix 8).

\section{Counselling for high-risk subjects}

After the in-person interview, high-risk subjects are advised with general recommendations for healthy lifestyle changes by trained cardiologists. The counselling includes the following eight general recommendations, 
given as needed to patients based on their in-person interview results: stick to a healthy, low-fat diet; engage in regular physical activity; lose weight; quit smoking; limit alcohol consumption; maintain a healthy daily routine with sufficient sleep; have a routine annual physical examination (eg, blood pressure, heart rate); and comply with all medication requirements. In addition, potential CVD patients are recommended to obtain further diagnoses and treatments. A list of the recommendations is included in online supplementary appendix 8 .

After counselling, all high-risk subjects are asked to set up a 1-month follow-up appointment.

\section{Follow-up of high-risk subjects}

To track changes in their lifestyles and risk factor statuses, high-risk subjects are followed-up after 1 month, either in a return clinic visit or by telephone interview. A return clinic visit includes physical measurements and a face-to-face interview. The physical measurements include blood pressure, weight, and an ECG using the same standard protocols applied in the screening phase. Results of the follow-up examination are recorded in a report (see online supplementary appendix 9). The face-to-face interview is administered by a trained interviewer to investigate the subject's survival status, hospitalisations, health-related lifestyle status (eg, smoking, alcohol use/misuse, physical activity, diet), ${ }^{37} 38$ and quality of life $^{40}$ (see online supplementary appendix 10). Telephone interviews are offered to any subjects who are unable to make a return clinic visit. A full list of variables examined is included in table 1 .

A written report on the results of the follow-up of high-risk subjects is given to each participant (see online supplementary appendix 9).

\section{Data management}

\section{Data handling and quality control}

Trained medical staff members enter all data from the questionnaires and health check-ups at each site into an off-line electronic data collection (EDC) system developed specifically for the pilot. To ensure the reliability and validity of the data, the off-line EDC system performs internal data checks to verify that the data being entered are complete and meet predefined data ranges and formats (see online supplementary appendix 11). The system displays a message warning users to correct or review data if they deviate from the predetermined data-checking rules. In addition, for each participant, the physical measurement data from the initial screening are entered into the off-line EDC system twice so that the system can verify their consistency.

At local sites, the data collected in the off-line EDC system are transferred to a central computer with internet access using an encrypted hard disk. Once transferred to the central computer, the data are then encrypted and confidentially stored in the NCCD.

A web-based project management platform was developed to monitor project progress and data quality. This platform also provides management support for the hospitals, staff members, equipment, sampling materials and funds used in the pilot.

\section{Data security and confidentiality}

All data, including health assessment results and questionnaires, are treated as protected information and are securely stored in an encrypted and password-protected database in the NCCD. This database can be accessed by only a limited number of approved staff members. At the local sites, all medical staff members must use their own passwords to log into the off-line EDC system. The passwords are used not only to ensure data security, but also to create an audit trail of all data entered or changed.

The data confidentiality policies (see online supplementary appendix 12) of the NCCD on data collection, storage and analysis have been strictly enforced in order to ensure the confidentiality of all personal information. The usage of the data is governed by the Research Guidance Committee, which consists of investigators from the NCCD and has been approved by an institutional review board.

\section{Quality assurance}

All local site medical staff members are trained in conducting blood pressure measurements, lipid blood tests, blood collection and sample processing, ECGs, ultrasound scans, face-to-face interviews and data entry. The local bureaus of quality and technical monitoring annually calibrate the site's electronic blood pressure monitor and rapid lipid analyser. In addition to the off-line EDC system's internal data-checking function, the validity of the collected data is verified monthly by searching for outliers in continuous data distributions, data with invalid and illogical values, and duplicate record entries. Once a potential error is found, data managers from the NCCD review the relevant records and resolve the problems (eg, correct invalid records, improve the data entry process, retrain local medical staff). When the data are being cleaned, outliers of measurement variables beyond the range of mean \pm 3 times SD are removed.

In addition, on-site monitoring by trained staff members from the NCCD is conducted at least once at each local site during the pilot. The monitoring examines each site's documentation completeness and staff compliance with recruitment, screening, physical measurements, sample collection and processing protocols.

\section{PRELIMINARY ANALYSIS}

As the pilot is still in progress, we used the first 0.1 million subjects screened (25000 in each province) to conduct preliminary data analysis. The current response rate of the initial screening participants was calculated using official residential records provided by each region. The mean values, prevalence rates, and SDs of the participants' 
Table 2 Characteristics of screened subjects

\begin{tabular}{|c|c|c|c|c|c|c|c|}
\hline & \multicolumn{2}{|c|}{ Male ( $\mathrm{N}=42$ 469) } & \multicolumn{2}{|c|}{ Female ( $\mathrm{N}=57$ 531) } & \multicolumn{2}{|c|}{ Total (N=100 000) } & \multirow[b]{2}{*}{ p Value } \\
\hline & $\mathrm{N}$ or mean & $\%$ or SD & $\mathrm{N}$ or mean & $\%$ or SD & $\mathbf{N}$ or mean & $\%$ or SD & \\
\hline Age (years) & & & & & & & $<0.001$ \\
\hline $40-49$ & 10454 & 24.62 & 14594 & 25.37 & 25048 & 25.05 & \\
\hline $50-59$ & 13651 & 32.14 & 19536 & 33.96 & 33187 & 33.19 & \\
\hline $60-69$ & 13993 & 32.95 & 18326 & 31.85 & 32319 & 32.32 & \\
\hline $70-75$ & 4256 & 10.02 & 4979 & 8.65 & 9235 & 9.24 & \\
\hline Total† & 57.06 & 9.27 & 56.59 & 10.68 & 56.79 & 10.11 & $<0.001$ \\
\hline Han nationality & 34838 & 82.03 & 46493 & 80.81 & 81331 & 81.33 & $<0.001$ \\
\hline Hukou status & & & & & & & $<0.001$ \\
\hline Non-agricultural & 12661 & 29.81 & 18652 & 32.42 & 31313 & 31.31 & \\
\hline Agricultural & 21716 & 51.13 & 29109 & 50.60 & 50825 & 50.83 & \\
\hline Unified Residency Hukou & 8088 & 19.04 & 9765 & 16.97 & 17853 & 17.85 & \\
\hline Do not have Hukou & 4 & 0.01 & 5 & 0.01 & 9 & 0.01 & \\
\hline Marital status & & & & & & & $<0.001$ \\
\hline Married with spouse & 40543 & 95.46 & 52244 & 90.81 & 92787 & 92.79 & \\
\hline Widowed, separated, divorced & 1379 & 3.25 & 4880 & 8.48 & 6259 & 6.26 & \\
\hline Never married & 311 & 0.73 & 68 & 0.12 & 379 & 0.38 & \\
\hline Unknown & 173 & 0.41 & 256 & 0.44 & 429 & 0.43 & \\
\hline Refuse to answer & 63 & 0.15 & 83 & 0.14 & 146 & 0.15 & \\
\hline Education & & & & & & & $<0.001$ \\
\hline Illiterate & 1283 & 3.02 & 4195 & 7.29 & 5478 & 5.48 & \\
\hline Less than primary school & 1201 & 2.83 & 2590 & 4.50 & 3791 & 3.79 & \\
\hline Primary school & 12837 & 30.23 & 18902 & 32.86 & 31739 & 31.74 & \\
\hline Middle school & 14757 & 34.75 & 18310 & 31.83 & 33067 & 33.07 & \\
\hline High school & 8508 & 20.03 & 9689 & 16.84 & 18197 & 18.20 & \\
\hline College or university & 3736 & 8.80 & 3681 & 6.40 & 7417 & 7.42 & \\
\hline Household income (Yuan/year) & & & & & & & $<0.001$ \\
\hline$<5000$ & 5426 & 12.78 & 7996 & 13.90 & 13422 & 13.42 & \\
\hline 5000-9999 & 2659 & 6.26 & 3888 & 6.76 & 6547 & 6.55 & \\
\hline $10000-19999$ & 7078 & 16.67 & 11418 & 19.85 & 18496 & 18.50 & \\
\hline $20000-50000$ & 15646 & 36.84 & 19369 & 33.67 & 35015 & 35.02 & \\
\hline$>50000$ & 5352 & 12.60 & 6260 & 10.88 & 11612 & 11.61 & \\
\hline Unknown & 1271 & 2.99 & 2198 & 3.82 & 3469 & 3.47 & \\
\hline Refuse to answer & 5037 & 11.86 & 6402 & 11.13 & 11439 & 11.44 & \\
\hline Current smoker & 16921 & 39.84 & 1503 & 2.61 & 18424 & 18.42 & $<0.001$ \\
\hline Alcohol drinker & & & & & & & $<0.001$ \\
\hline Never & 22148 & 52.15 & 51703 & 89.87 & 73851 & 73.85 & \\
\hline Monthly or less & 3194 & 7.52 & 2293 & 3.99 & 5487 & 5.49 & \\
\hline 2-4 times a month & 5453 & 12.84 & 1487 & 2.58 & 6940 & 6.94 & \\
\hline 2-3 times a week & 11337 & 26.69 & 1405 & 2.44 & 12742 & 12.74 & \\
\hline
\end{tabular}

baseline characteristics were calculated using $\chi^{2}$ tests for comparison between men and women. The prevalence rates of high-risk subjects were also calculated using $\chi^{2}$ tests for comparison among the four provinces. Preliminary data cleaning was conducted in order to remove missing values and outliers from the dataset. Data analysis was performed using the SPSS V.18.0 software package.

\section{RESULTS}

Demographic characteristics of participants

Of the 0.1 million participants aged $40-75$ years in the present analysis, the estimated response rate was $32.1 \%$ for the three provinces (Liaoning, Jilin and Zhejiang) that have completed the initial screening. The follow-up rate was $74.3 \%$. The demographic characteristics of the participants are shown in table 2. Among the 0.1 million participants, $42.5 \%$ were men, $50.8 \%$ were registered in the agricultural Hukou, and the mean \pm SD age was 56.8 \pm 10.1 years. Nearly all participants were married, and the proportion without a spouse (widowed, separated or divorced) was more than twice as high for women as for men $(8.5 \%$ vs $3.3 \%)$. About half of the participants had at least 9 years of formal education. The prevalence of current smokers was significantly higher among men than women $(39.8 \%$ vs $2.6 \%, \mathrm{p}<0.001)$. The majority of participants reported that they had never drunk alcohol. 
A significant difference in prevalence of regular alcohol drinkers was observed between men and women $(26.7 \%$ vs $2.4 \%, \mathrm{p}<0.001)$.

\section{Baseline anthropometric parameters and medical history} of the participants

Table 3 shows the anthropometric parameters and medical history of the first 0.1 million participants screened with valid baseline data. The mean BMI was $24.3 \mathrm{~kg} / \mathrm{m}^{2}$, with $37.8 \%$ qualifying as overweight or obese $\left(\geq 25 \mathrm{~kg} / \mathrm{m}^{2}\right)$. The prevalence of hypertension ( $\mathrm{SBP} \geq 140 \mathrm{~mm} \mathrm{Hg}$ or $\mathrm{DBP} \geq 90 \mathrm{~mm} \mathrm{Hg})$ was $47.6 \%$ in men and $43.9 \%$ in women. The proportion of participants reporting a history of hypertension was $20.9 \%$, which was higher than that of any other chronic disease, and the proportion of those reporting hypertension was significantly higher among women than men $(21.9 \%$ vs $19.4 \%, \mathrm{p}<0.001)$. The proportion of participants who reported having a history of diabetes was $5.2 \%$ in men and $6.2 \%$ in women.

\section{Prevalence of subjects with high CVD risk}

The prevalence rates in each province of subjects with high CVD risk is shown in table 4 . Overall, $26.9 \%$ of participants were identified as high-risk subjects. The proportion varied significantly between the four provinces, ranging from $21.1 \%$ to $35.8 \%(\mathrm{p}<0.001)$. Of all the identified high-risk subjects, $20.8 \%$ had a prior history of CVD, $68.1 \%$ had high blood pressure, $25.3 \%$ had dyslipidaemia, and $26.1 \%$ had a 10 -year CVD risk $\geq 20 \%$. Again, each of these proportions varied significantly by area $(\mathrm{p}<0.001)$.

\section{DISCUSSION}

This pilot project is the first large-scale population-based screening initiative in China aimed at identifying subjects at high risk of CVD and collecting detailed information and biospecimens as part of a precision medicine project. Implemented using a rigorous methodological design and standardised health data collection methods, this public health effort may serve a variety of important purposes. First, it can make a tangible contribution to population health through its screening and intervention function. Second, it can be used as a research-grade database for future precision medicine investigation of population risk factors and outcomes of CVD. In addition, the pilot will expand current knowledge regarding China's growing epidemic of high-risk CVD, which will prove invaluable as China continues its epidemiological transition to a nation

Table 3 Anthropometric variables and medical history of screened subjects

\begin{tabular}{|c|c|c|c|c|c|c|c|}
\hline \multirow[b]{2}{*}{ Variable } & \multicolumn{2}{|c|}{ Male ( $\mathrm{N}=42$ 469) } & \multicolumn{2}{|c|}{ Female ( $\mathrm{N}=57531)$} & \multicolumn{2}{|c|}{ Total $(\mathrm{N}=10000)$} & \multirow[b]{2}{*}{ p Value ${ }^{\star}$} \\
\hline & $\mathbf{N}$ or mean & $\%$ or SD & $\mathbf{N}$ or mean & $\%$ or SD & $\mathbf{N}$ or mean & $\%$ or SD & \\
\hline Height (cm) & 166.81 & 6.91 & 156.22 & 6.42 & 160.72 & 8.45 & $<0.001$ \\
\hline Weight (kg) & 68.22 & 10.34 & 59.21 & 9.39 & 63.03 & 10.77 & $<0.001$ \\
\hline BMI $\left(\mathrm{kg} / \mathrm{m}^{2}\right)$ & 24.46 & 3.05 & 24.23 & 3.36 & 24.33 & 3.24 & \\
\hline$<18.5$ & 583 & $1.37 \%$ & 1449 & $2.52 \%$ & 2032 & $2.03 \%$ & \\
\hline $18.5-24.9$ & 25237 & $59.42 \%$ & 34903 & $60.67 \%$ & 60140 & $60.14 \%$ & \\
\hline $25.0-29.9$ & 14656 & $34.51 \%$ & 17984 & $31.26 \%$ & 32640 & $32.64 \%$ & \\
\hline$\geq 30.0$ & 1974 & $4.65 \%$ & 3195 & $5.55 \%$ & 5169 & $5.17 \%$ & \\
\hline $\mathrm{SBP}(\mathrm{mm} \mathrm{Hg})$ & 140.53 & 19.33 & 138.96 & 20.54 & 139.63 & 20.05 & $<0.001$ \\
\hline $\mathrm{DBP}(\mathrm{mm} \mathrm{Hg})$ & 82.91 & 10.78 & 80.10 & 10.80 & 81.29 & 10.88 & $<0.001$ \\
\hline High blood pressure & 20193 & $47.55 \%$ & 25275 & $43.93 \%$ & 45468 & $45.47 \%$ & $<0.001$ \\
\hline $\mathrm{TC}(\mathrm{mmol} / \mathrm{L})$ & 4.65 & 1.02 & 5.01 & 1.12 & 4.86 & 1.09 & $<0.001$ \\
\hline $\mathrm{TG}(\mathrm{mmol} / \mathrm{L})$ & 1.88 & 1.17 & 2.02 & 1.19 & 1.96 & 1.18 & $<0.001$ \\
\hline HDL-C (mmol/L) & 1.44 & 0.46 & 1.58 & 0.45 & 1.52 & 0.46 & $<0.001$ \\
\hline LDL-C (mmol/L) & 2.50 & 0.84 & 2.65 & 0.90 & 2.58 & 0.88 & $<0.001$ \\
\hline \multicolumn{8}{|l|}{ Medical history } \\
\hline Hypertension & 8254 & $19.44 \%$ & 12602 & $21.90 \%$ & 20856 & $20.86 \%$ & $<0.001$ \\
\hline Diabetes & 2197 & $5.17 \%$ & 3536 & $6.15 \%$ & 5733 & $5.73 \%$ & $<0.001$ \\
\hline $\mathrm{Ml}$ & 512 & $1.21 \%$ & 486 & $0.84 \%$ & 998 & $1.00 \%$ & $<0.001$ \\
\hline $\mathrm{PCl}$ & 272 & $0.64 \%$ & 143 & $0.25 \%$ & 415 & $0.42 \%$ & $<0.001$ \\
\hline CABG & 39 & $0.09 \%$ & 31 & $0.05 \%$ & 70 & $0.07 \%$ & 0.025 \\
\hline Stroke & 2031 & $4.78 \%$ & 2466 & $4.29 \%$ & 4497 & $4.50 \%$ & $<0.001$ \\
\hline Haemorrhage stroke & 1604 & $3.78 \%$ & 2016 & $3.50 \%$ & 3620 & $3.62 \%$ & \\
\hline Ischaemic stroke & 194 & $0.46 \%$ & 161 & $0.28 \%$ & 355 & $0.36 \%$ & \\
\hline
\end{tabular}

Values are $\mathrm{n}(\%)$ or mean (SD) as indicated.

${ }^{*} \chi^{2}$ test for proportion and two-tailed $t$ test (or $t^{\prime}$ test if equal variances not assumed) for means, $\alpha=0.05$.

tHigh blood pressure: $\mathrm{SBP} \geq 140 \mathrm{mmHg}$ or $\mathrm{DBP} \geq 90 \mathrm{mmHg}$.

BMI, body mass index; CABG, coronary artery bypass grafting; DBP, diastolic blood pressure; HDL-C, high-density lipoprotein cholesterol; LDL-C, low-density lipoprotein cholesterol; MI, myocardial infarction; $\mathrm{PCl}$, percutaneous coronary intervention; SBP, systolic blood pressure; TC, total cholesterol; TG, triglyceride. 
Table 4 Prevalence of high-risk subjects of all and each type of high-risk criterion

\begin{tabular}{|c|c|c|c|c|c|c|c|c|c|c|c|}
\hline \multirow[b]{2}{*}{ Criterion } & \multicolumn{2}{|c|}{ Liaoning } & \multicolumn{2}{|l|}{ Jilin } & \multicolumn{2}{|c|}{ Zhejiang } & \multicolumn{2}{|c|}{ Guangxi } & \multicolumn{2}{|l|}{ Total } & \multirow[b]{2}{*}{$\begin{array}{l}p \\
\text { Value }\end{array}$} \\
\hline & $\mathbf{N}$ & $\begin{array}{l}\text { Per } \\
\text { cent }\end{array}$ & $\mathbf{N}$ & $\begin{array}{l}\text { Per } \\
\text { cent }\end{array}$ & $\mathbf{N}$ & $\begin{array}{l}\text { Per } \\
\text { cent }\end{array}$ & $\mathbf{N}$ & $\begin{array}{l}\text { Per } \\
\text { cent }\end{array}$ & $\mathbf{N}$ & $\begin{array}{l}\text { Per } \\
\text { cent }\end{array}$ & \\
\hline $\begin{array}{l}\text { Total CVD high-risk } \\
\text { subjects }\end{array}$ & 6712 & 26.85 & 8961 & 35.84 & 5269 & 21.08 & 5957 & 23.83 & 26899 & 26.90 & $<0.001$ \\
\hline CVD disease & 1225 & 18.25 & 3061 & 34.16 & 347 & 6.59 & 968 & 16.25 & 5601 & 20.82 & $<0.001$ \\
\hline High blood pressure & 4898 & 72.97 & 5774 & 64.43 & 4156 & 78.88 & 3481 & 58.44 & 18309 & 68.07 & $<0.001$ \\
\hline Dyslipidaemia & 1569 & 23.38 & 1857 & 20.72 & 1045 & 19.83 & 2340 & 39.28 & 6811 & 25.32 & $<0.001$ \\
\hline WHO risk $\geq 20 \%$ & 1817 & 27.07 & 2125 & 23.71 & 1450 & 27.52 & 1614 & 27.09 & 7006 & 26.05 & $<0.001$ \\
\hline No risk & 18288 & 73.15 & 16039 & 64.16 & 19731 & 78.92 & 19043 & 76.17 & 73101 & 73.10 & \\
\hline
\end{tabular}

marked by a widespread rise in non-communicable diseases.

To counteract the expected upswing of CVD events in China in the years to come, national CVD prevention and intervention initiatives are urgently needed. This pilot is a novel, large-scale, longitudinal project on the modern Chinese epidemic of CVD. The project is designed to address the nation's knowledge gap regarding CVD and better inform future efforts in CVD prevention.

Previous large-scale, Chinese-population-based CVD studies have been limited to determining the prevalence of CVD risk factors without actually identifying high-risk subjects and comprehensively assessing their cardiovascular health. ${ }^{37} 394243$ Only one CVD study has identified a high-risk CVD population, but this study was hindered by the fact that it was cross-sectional and limited to rural residents in only one province. ${ }^{30}$ Therefore, outside of this pilot project, there have been no other longitudinal, large-scale studies that use risk stratification to detect high-risk populations in China, and then conduct detailed health assessments and follow-up on them. In addition to its large scale, our pilot employed standardised, efficient and selfmonitoring EDC on a wide range of information relevant to cardiovascular health and other disease, involved the mass storage of biospecimens, and included large-scale follow-up using clinical trial methodology. Thus, our pilot is unique in its ability to provide original and accurate data for characterising populations with high CVD risk through a highly efficient process. It is designed to answer research questions related to sociodemographics, biology, health behaviours, health trajectories, and the relationship between CVD risk factors and outcomes in high-risk populations. It will allow policymakers and academics to produce evidence-based research to inform future approaches to CVD prevention and intervention and may serve as a possible model for the development of similar projects in other countries.

In addition to informing future primary and secondary CVD prevention programmes, this pilot project has a wide range of public health implications. The data collected from the pilot could contribute to the establishment of a biobank aimed at promoting basic research that could provide essential knowledge regarding CVDs and other conditions. The pilot's wide range of data on biology, health behaviours and sociodemographics will serve as a powerful database for future investigators of CVD interested in topics, such as the complex interaction between a patient's lifestyle and genetic factors, that can act as a determinant of CVD. The pilot is also relevant to precision medicine, as it will allow better understanding of the genetic and non-genetic causes of CVD across a variety of conditions. In addition, the potential uses of the pilot extend beyond CVD. The biosamples and health behaviour data collected in the pilot may be applied to the study of effects of certain risk factors (eg, smoking, blood pressure, cholesterol) on other diseases. Lastly, the health assessment and data collection methodologies developed in the pilot have the potential to inform future strategies for the prevention and management of other noncommunicable diseases.

The pilot will ultimately be implemented as a nationwide CVD screening project between 2015 and 2020, and may serve as a model for the development of similar projects in other countries. In the next phases, random sampling methods will be applied, and biosamples will be collected at the initial screening, which may promote future establishment of a biobank for millions of people in China. Considering the rising incidence of CVD in younger persons, we also plan to expand the current age range to $35-75$ years. In addition, a long-term (1-year) follow-up of the pilot's high-risk subjects will be conducted later this year to collect important insights into the optimal development methods of a sustainable CVD prevention and intervention project. The follow-up will focus on several areas, including the efficacy of longterm behavioural health monitoring and risk factor modification.

Along with the rapid economic growth, urbanisation and westernised lifestyle, the prevalence of obesity, diabetes and hypertension in China has been dramatically 
expanding. Our preliminary results showed that the prevalence of subjects with high CVD risk was $26.9 \%$. According to the results, the prevalence of overweight, obesity and hypertension all increased significantly from their respective values reported in the 2002 China National Nutrition and Health Survey. ${ }^{45}$ However, our data's estimated prevalence of current smokers has decreased from that reported in the 1996 National Prevalence Survey ${ }^{46}$ The proportion of subjects who reported having a history of diabetes remained similar to the results from the International Collaborative Study of Cardiovascular Disease in Asia (InterAsia). ${ }^{43}$ Our results for blood pressure measurements and the combined prevalence of overweight and obesity were similar to those found in the China Kadoorie Biobank Study of 0.5 million people. ${ }^{37}$ However, previous populationbased studies conducted in China tended to include younger subjects than our sample. Compared with American adults, the combined prevalence of overweight and obesity based on our data was much lower in Chinese, ${ }^{47}$ but the prevalence of hypertension was almost twice as high in China as that reported in the US study. ${ }^{48}$

The pilot is limited in three ways. First, the response rate for the pilot was not high-about $32 \%$. The response rate may have been driven down by the fact that many of the participants with a rural Hukou (about half of the total sample of people) live and work in cities, which might make it more difficult for them to participate in the study. In addition, participation was entirely voluntary, which may also have driven down the response rate. However, because of its large size, we believe that our sample is large enough to capture the full diversity of the Chinese population. Our response rate is also consistent with and a modest improvement over that seen in the China Kadoorie Biobank, a study that employed similar recruitment strategies. ${ }^{37}$ And it is a significant improvement over the low response rate seen in Europe for similar studies, such as the UK Biobank study $(5-10 \%)^{49}$ and a study that constructed a nationwide biobank in Estonia $(\sim 5 \%) .{ }^{50}$ Second, since the pilot was conducted primarily to test the feasibility of a large-scale screening programme, a convenient rather than nationally representative sample was used to ensure rapid and sizable recruitment. However, in the full phase, random sampling will be used to select a nationally representative sample. Third, no medication or lifestyle interventions were offered to high-risk subjects in the pilot phase. Instead, only general recommendations on lifestyle changes were provided, as it would be unrealistic to properly evaluate the efficacy of any such intervention after only a 1-month follow-up. Nevertheless, these limitations are minor considering the scope of the pilot project. The project's use of a rigorous methodological design and thorough data collection methodologies ensure its ability to act as a resource for future investigation into the prevention of CVD.
As the incidence rate of CVD increases nationwide, it is critical for China to acknowledge, contain and counteract the threat that CVD poses to the nation's longterm health and economic well-being. As the first large-scale screening initiative of its kind, the pilot will provide a powerful first step for China in this process. This pilot will demonstrate the feasibility of conducting a large-scale screening effort with research-grade methods. Consequently, it will lay the foundation for future national CVD prevention and intervention studies and has the ability to promote meaningful national efforts to improve cardiovascular, and overall, health.

\section{Author affiliations}

${ }^{1}$ National Clinical Research Center of Cardiovascular Diseases, State Key Laboratory of Cardiovascular Disease, Fuwai Hospital, National Center for Cardiovascular Diseases, Chinese Academy of Medical Sciences and Peking Union Medical College, Beijing, People's Republic of China

${ }^{2}$ Center for Outcomes Research and Evaluation, Yale-New Haven Hospital, New Haven, Connecticut, USA

${ }^{3}$ Department of Health Policy and Management, Yale School of Public Health, New Haven, Connecticut, USA

${ }^{4}$ Section of Cardiovascular Medicine, Robert Wood Johnson Clinical Scholars Program, Department of Internal Medicine, Yale University School of Medicine, New Haven, Connecticut, USA

Acknowledgements We appreciate the multiple contributions made by project teams at the National Center for Cardiovascular Diseases and the Yale-New Haven Hospital Center for Outcomes Research and Evaluation in the realms of project design and operations. We appreciate the advice from Weiwei Chen, Yang Yang, Zhengming Chen, Yu Guo, Ralph Horwitz and Sharon-Lise Normand. We thank Xingguo Sun and Hao Wang for their contributions on ECG and cardiac and carotid ultrasound examination protocol, respectively. We thank Yuan Lu, Emily Gudbranson and Hui Qu for their contributions to the manuscript. We thank all provincial and regional staff, officers in the four provinces (Liaoning, Jilin, Zhejiang and Guangxi), for their collection of data and biosamples. We thank all the subjects for their participation. We are grateful for the support provided by the Chinese government.

Contributors HMK and LJ conceived of the screening for subjects with high CVD risk and take responsibility for all aspects of it. LJ, HMK, JL and CW designed the project. JL and SX wrote the first draft of the article, with further contributions from NSD, CW, LL, HMK and LJ. CW and JL performed statistical analysis. All authors approved the final version of the article.

Funding This project is a major public health service project supported by the Ministry of Finance of China and National Health and Family Planning Commission of China, and the National Key Technology R\&D Program (2013BAI09B01) from the Ministry of Science and Technology of China. HMK is supported by grant U01 HL105270-05 (Center for Cardiovascular Outcomes Research at Yale University) from the National Heart, Lung, and Blood Institute. The sponsors had no role in the conduct of the study, the collection, management, analysis and interpretation of the data, or the preparation or approval of the manuscript.

Competing interests HMK works under contract with the Centers for Medicare \& Medicaid Services to develop and maintain performance measures, is chair of a cardiac scientific advisory board for UnitedHealth, and is the recipient of research grants from Medtronic and Johnson \& Johnson through Yale University.

\section{Patient consent Obtained.}

Ethics approval The central ethics committee at the National Center for Cardiovascular Disease (NCCD) approved the pilot (Trial Registration Number NCT02536456).

Provenance and peer review Not commissioned; externally peer reviewed. 
Open Access This is an Open Access article distributed in accordance with the Creative Commons Attribution Non Commercial (CC BY-NC 4.0) license, which permits others to distribute, remix, adapt, build upon this work noncommercially, and license their derivative works on different terms, provided the original work is properly cited and the use is non-commercial. See: http:// creativecommons.org/licenses/by-nc/4.0/

\section{REFERENCES}

1. National Research Council. Toward precision medicine: building a knowledge network for biomedical research and a new taxonomy of disease. Washington DC: National Academies Press, 2011.

2. $\mathrm{Bi} \mathrm{Y}$, Jiang $\mathrm{Y}, \mathrm{He} \mathrm{J}$, et al. Status of cardiovascular health in Chinese adults. J Am Coll Cardiol 2015;65:1013-25.

3. He J, Gu D, Chen J, et al. Premature deaths attributable to blood pressure in China: a prospective cohort study. Lancet 2009;374:1765-72.

4. Odegaard AO, Koh WP, Yuan JM, et al. Western-style fast food intake and cardiometabolic risk in an Eastern country. Circulation 2012;126:182-8.

5. He J, Gu D, Wu X, et al. Major causes of death among men and women in China. N Engl J Med 2005;353:1124-34.

6. Popkin BM. Synthesis and implications: China's nutrition transition in the context of changes across other low- and middle-income countries. Obes Rev 2014;15(Suppl 1):60-7.

7. Wang $X-Q$, Chen $P$-J. Population ageing challenges health care in China. Lancet 2014;383:870.

8. Moran A, Gu D, Zhao D, et al. Future cardiovascular disease in China: Markov model and risk factor scenario projections from the coronary heart disease policy model-china. Circ Cardiovasc Qual Outcomes 2010;3:243-52.

9. Wang S, Marquez P, Langenbrunner J. Toward a healthy and harmonious life in China: stemming the rising tide of non-communicable diseases. Washington: The World Bank, 2011.

10. Eckel RH, Jakicic JM, Ard JD, et al. 2013 AHA/ACC guideline on lifestyle management to reduce cardiovascular risk: a report of the American College of Cardiology/American Heart Association Task Force on practice guidelines. Circulation 2014;129(25_Suppl_2): S76-99.

11. Chinese Society of Cardiology of Chinese Medical Association, Editorial Board of Chinese Journal of Cardiology. [Chinese guidelines for prevention of cardiovascular diseases]. Zhonghua Xin Xue Guan Bing Za Zhi 2011;39:3-22.

12. Scandinavian Simvastatin Survival Study Group. Randomised trial of cholesterol lowering in 4444 patients with coronary heart disease: the Scandinavian Simvastatin Survival Study (4S). Lancet 1994;344:1383-9.

13. Sacks FM, Pfeffer MA, Moye LA, et al. The effect of pravastatin on coronary events after myocardial infarction in patients with average cholesterol levels. Cholesterol and Recurrent Events Tria investigators. N Engl J Med 1996;335:1001-9.

14. Tonkin A, Aylward P, Colquhoun D, et al. Prevention of cardiovascular events and death with Pravastatin in patients with coronary heart disease and a broad range of initial cholesterol levels. N Engl J Med 1998;339:1349-57.

15. Baigent C, Keech A, Kearney PM, et al., Cholesterol Treatment Trialists' (CTT) Collaborators. Efficacy and safety of cholesterol-lowering treatment: prospective meta-analysis of data from 90,056 participants in 14 randomised trials of statins. Lancet 2005;366:1267-78.

16. The ALLHAT Officers and Coordinators for the ALLHAT Collaborative Research Group. Major cardiovascular events in hypertensive patients randomized to doxazosin vs chlorthalidone. The Antihypertensive and Lipid-Lowering Treatment to Prevent Heart Attack Trial (ALLHAT). JAMA 2000;283:1967-75.

17. Psaty BM, Lumley T, Furberg CD, et al. Health outcomes associated with various antihypertensive therapies used as first-line agents: a network meta-analysis. JAMA 2003;289:2534-44.

18. Clair C, Rigotti NA, Porneala B, et al. Association of smoking cessation and weight change with cardiovascular disease among adults with and without diabetes. JAMA 2013;309:1014-21.

19. Kodama S, Tanaka S, Heianza, Y, et al. Association between physical activity and risk of all-cause mortality and cardiovascular disease in patients with diabetes: a meta-analysis. Diabetes Care 2013;36:471-9.

20. Shortreed SM, Peeters A, Forbes AB. Estimating the effect of long-term physical activity on cardiovascular disease and mortality evidence from the Framingham Heart Study. Heart 2013;99:649-54

21. Cook NR, Cutler JA, Obarzanek E, et al. Long term effects of dietary sodium reduction on cardiovascular disease outcomes: observational follow-up of the trials of hypertension prevention (TOHP). BMJ 2007;334:885-8.

22. Yu-Poth S, Zhao G, Etherton T, et al. Effects of the National Cholesterol Education Program's Step I and Step II dietary intervention programs on cardiovascular disease risk factors: a meta-analysis. Am J Clin Nutr 1999;69:632-46.

23. Ness AR, Powles JW. Fruit and vegetables, and cardiovascular disease: a review. Int J Epidemiol 1997;26:1-13.

24. Berger JS, Jordan CO, Lloyd-Jones D, et al. Screening for cardiovascular risk in asymptomatic patients. J Am Coll Cardio 2010;55:1169-77.

25. American College of Cardiology. Matching the intensity of risk factor management with the hazard for coronary disease events. J Am Coll Cardiol 1995;96:957-1047

26. Greenland P, Alpert JS, Beller GA et al. 2010 ACCF/AHA guideline for assessment of cardiovascular risk in asymptomatic adults: a report of the American College of Cardiology Foundation/American Heart Association Task Force on Practice Guidelines. Circulation 2010;122:e584-636.

27. Xu Y, Wang L, He J, et al. Prevalence and control of diabetes in Chinese adults. JAMA 2013;310:948-59.

28. Wang $\mathrm{S}, \mathrm{Xu} \mathrm{L}$, Jonas JB, et al. Prevalence and associated factors of dyslipidemia in the adult Chinese population. PLOS ONE 2011;6: e17326.

29. Wang $\mathrm{H}$, Zhang $\mathrm{X}$, Zhang J, et al. Factors associated with prevalence, awareness, treatment and control of hypertension among adults in Southern China: a community-based cross-sectional survey. PLOS ONE 2013;8:e62469.

30. Wei X, Zou G, Yin J, et al. Characteristics of high risk people with cardiovascular disease in Chinese rural areas: clinical indictors, disease patterns and drug treatment. PLOS ONE 2013;8: e54169.

31. Dharmarajan K, Li J, Li X, et al. The China Patient-Centered Evaluative Assessment of Cardiac Events (China PEACE) retrospective study of acute myocardial infarction: study design Circ Cardiovasc Qual Outcomes 2013;6:732-40.

32. Li J, Dharmarajan K, Li X, et al. Protocol for the China PEACE (Patient-centered Evaluative Assessment of Cardiac Events) retrospective study of coronary catheterisation and percutaneous coronary intervention. BMJ Open 2014;4:e004595.

33. Collins FS, Varmus $\mathrm{H}$. A new initiative on precision medicine. $N$ Engl J Med 2015;372:793-5.

34. World Health Organization. Prevention of cardiovascular disease. Guidelines for assessment and management of cardiovascular risk. Geneva: WHO, 2007.

35. World Health Organization/International Society of Hypertension. WHO/ISH Risk prediction charts for 14 WHO epidemiological sub-regions. Geneva: WHO, 2007.

36. Bidgood WD, Horii SC, Prior FW, et al. Understanding and using DICOM, the data interchange standard for biomedical imaging. J Am Med Inform Assoc 1997;4:199-212.

37. Chen Z, Chen J, Collins R, et al. China Kadoorie Biobank of 0.5 million people: survey methods, baseline characteristics and long-term follow-up. Int J Epidemiol 2011;40: 1652-66.

38. Li Q, Babor TF, Hao W, et al. The Chinese translations of Alcohol Use Disorders Identification Test (AUDIT) in China: a systematic review. Alcohol Alcohol 2011;46:416-23.

39. Zhao Y, Strauss J, Yang G, et al. China Health and Retirement Longitudinal Study-2011-2012 national baseline users' guide. Beijing: National School of Development, Peking University, 2013.

40. Wang HM, Patrick DL, Edwards TC, et al. Validation of the EQ-5D in a general population sample in urban China. Qual Life Res 2012;21:155-60.

41. Strauss J, Lei X, Park A, et al. Health outcomes and socio-economic status among the elderly in Gansu and Zhejiang Provinces, China: evidence from the CHARLS pilot. J Popul Ageing 2010;3:111-42.

42. Yang ZJ, Liu J, Ge JP, et al. Prevalence of cardiovascular disease risk factor in the Chinese population: the 2007-2008 China National Diabetes and Metabolic Disorders Study. Eur Heart $J$ 2012;33:213-20.

43. Gu D, Gupta A, Muntner P, et al. Prevalence of cardiovascular disease risk factor clustering among the adult population of China: results from the International Collaborative Study of Cardiovascular Disease in Asia (InterAsia). Circulation 2005;112:658-65.

44. Wang $Y, M i J$, Shan $X Y$, et al. Is China facing an obesity epidemic and the consequences? The trends in obesity and chronic disease in China. Int J Obes (Lond) 2007;31:177-88. 
45. Wu Y, Huxley R, Li L, et al. Prevalence, awareness, treatment, and control of hypertension in China: data from the China National Nutrition and Health Survey 2002. Circulation 2008;118:2679-86.

46. Yang G, Fan L, Tan J, et al. Smoking in China: findings of the 1996 National Prevalence Survey. JAMA 1999;282:1247-53.

47. Flegal KM, Carroll MD, Kit BK, et al. Prevalence of obesity and trends in the distribution of body mass index among US adults, 1999-2010. JAMA 2012;307:491-7.
48. Egan BM, Zhao Y, Axon RN. US trends in prevalence, awareness, treatment, and control of hypertension, 1988-2008. JAMA 2010;303:2043-50.

49. Manolio TA, Weis BK, Cowie CC, et al. New models for large prospective studies: is there a better way? Am J Epidemiol 2012;175:859-66.

50. Leitsalu L, Haller T, Esko T, et al. Cohort profile: Estonian Biobank of the Estonian Genome Center, University of Tartu. Int J Epidemiol 2015:44:1137-47. 
Erratum: Protocol for the China PEACE (Patient-centered Evaluative Assessment of Cardiac Events) Million Persons Project pilot

Lu J, Xuan S, Downing NS, et al. Protocol for the China PEACE (Patient-centered Evaluative Assessment of Cardiac Events) Million Persons Project pilot. BMJ Open 2015;5:e010200. There is an error in the last two rows of table 3. The number and percent of haemorrhage stroke and ischaemic stroke were reversed in total and gender subgroups. The correct table 3 is given below.

\begin{tabular}{|c|c|c|c|c|c|c|}
\hline & \multicolumn{2}{|c|}{ Male ( $\mathrm{N}=42469)$} & \multicolumn{2}{|c|}{ Female $(\mathrm{N}=57531)$} & \multicolumn{2}{|c|}{ Total $(\mathrm{N}=10000)$} \\
\hline & $\begin{array}{l}\mathbf{N} \text { or } \\
\text { mean }\end{array}$ & $\begin{array}{l}\% \text { or } \\
\text { SD }\end{array}$ & $\begin{array}{l}\mathbf{N} \text { or } \\
\text { mean }\end{array}$ & $\begin{array}{l}\mathbf{N} \text { or } \\
\text { mean }\end{array}$ & $\begin{array}{l}\% \text { or } \\
\text { SD }\end{array}$ & $\begin{array}{l}\mathrm{N} \text { or } \\
\text { mean }\end{array}$ \\
\hline Height (cm) & 166.81 & 6.91 & 156.22 & 6.42 & 160.72 & 8.45 \\
\hline Weight (kg) & 68.22 & 10.34 & 59.21 & 9.39 & 63.03 & 10.77 \\
\hline BMI $\left(\mathrm{kg} / \mathrm{m}^{2}\right)$ & 24.46 & 3.05 & 24.23 & 3.36 & 24.33 & 3.24 \\
\hline$<18.5$ & 583 & $1.37 \%$ & 1449 & $2.52 \%$ & 2032 & $2.03 \%$ \\
\hline $18.5-24.9$ & 25237 & $59.42 \%$ & 34903 & $60.67 \%$ & 60140 & $60.14 \%$ \\
\hline $25.0-29.9$ & 14656 & $34.51 \%$ & 17984 & $31.26 \%$ & 32640 & $32.64 \%$ \\
\hline$\geq 30.0$ & 1974 & $4.65 \%$ & 3195 & $5.55 \%$ & 5169 & $5.17 \%$ \\
\hline SBP (mm Hg) & 140.53 & 19.33 & 138.96 & 20.54 & 139.63 & 20.05 \\
\hline DBP (mm Hg) & 82.91 & 10.78 & 80.10 & 10.80 & 81.29 & 10.88 \\
\hline $\begin{array}{l}\text { High blood } \\
\text { pressure }^{\star}\end{array}$ & 20193 & $47.55 \%$ & 25275 & $43.93 \%$ & 45468 & $45.47 \%$ \\
\hline TC (mmol/L) & 4.65 & 1.02 & 5.01 & 1.12 & 4.86 & 1.09 \\
\hline $\mathrm{TG}(\mathrm{mmol} / \mathrm{L})$ & 1.88 & 1.17 & 2.02 & 1.19 & 1.96 & 1.18 \\
\hline HDL (mmol/L) & 1.44 & 0.46 & 1.58 & 0.45 & 1.52 & 0.46 \\
\hline LDL (mmol/L) & 2.50 & 0.84 & 2.65 & 0.90 & 2.58 & 0.88 \\
\hline \multicolumn{7}{|l|}{ Medical history } \\
\hline Hypertension & 8254 & $19.44 \%$ & 12602 & $21.90 \%$ & 20856 & $20.86 \%$ \\
\hline Diabetes & 2197 & $5.17 \%$ & 3536 & $6.15 \%$ & 5733 & $5.73 \%$ \\
\hline $\begin{array}{l}\text { Myocardial } \\
\text { infarction }\end{array}$ & 512 & $1.21 \%$ & 486 & $0.84 \%$ & 998 & $1.00 \%$ \\
\hline $\mathrm{PCl}$ & 272 & $0.64 \%$ & 143 & $0.25 \%$ & 415 & $0.42 \%$ \\
\hline CABG & 39 & $0.09 \%$ & 31 & $0.05 \%$ & 70 & $0.07 \%$ \\
\hline Stroke & 2031 & $4.78 \%$ & 2466 & $4.29 \%$ & 4497 & $4.50 \%$ \\
\hline Hemorrhage stroke & 194 & $0.46 \%$ & 161 & $0.28 \%$ & 355 & $0.36 \%$ \\
\hline Ischemic stroke & 1604 & $3.78 \%$ & 2016 & $3.50 \%$ & 3620 & $3.62 \%$ \\
\hline
\end{tabular}

Values are $\mathrm{n}(\%)$ or mean (SD) as indicated.

${ }^{*} \chi^{2}$ test for proportion and two-tailed $t$ test (or $t$ ' test if equal variances not assumed) for means, $\alpha=0.05$.

†High blood pressure: SBP $\geq 140 \mathrm{~mm} \mathrm{Hg}$ or DBP $\geq 90 \mathrm{~mm} \mathrm{Hg}$

$\mathrm{BMI}$, body mass index; $\mathrm{CABG}$, coronary artery bypass grafting; DBP, diastolic blood pressure;

HDL-C, high-density lipoprotein cholesterol; LDL-C, low-density lipoprotein cholesterol;

$\mathrm{MI}$, myocardial infarction; $\mathrm{PCl}$, percutaneous coronary intervention; SBP, systolic blood pressure;

TC, total cholesterol; TG, triglyceride.

Open Access This is an Open Access article distributed in accordance with the Creative Commons Attribution Non Commercial (CC BY-NC 4.0) license, which permits others to distribute, remix, adapt, build upon this work noncommercially, and license their derivative works on different terms, provided the original work is properly cited and the use is non-commercial. See: http://creativecommons.org/licenses/by-nc/4.0/

BMJ Open 2016;6:e010200corr1. doi:10.1136/bmjopen-2015-010200corr1

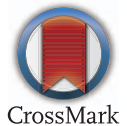

